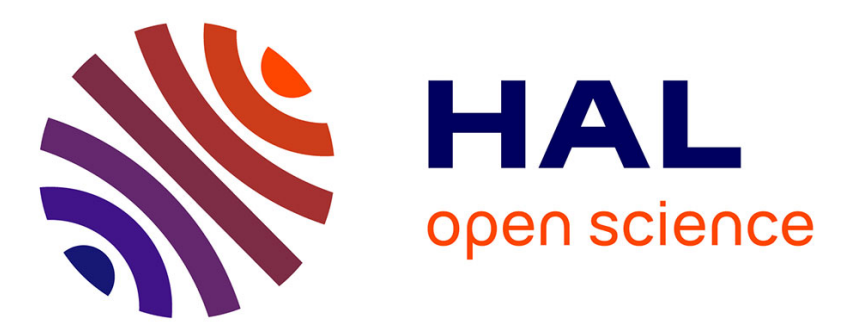

\title{
Etude préliminaire de l'influence de la fertilisation sur la qualité du bois de chêne
}

\author{
Hubert Polge
}

\section{To cite this version:}

Hubert Polge. Etude préliminaire de l'influence de la fertilisation sur la qualité du bois de chêne. Revue forestière française, 1975, 27 (3), pp.201-208. 10.4267/2042/20937 . hal-03395497

\section{HAL Id: hal-03395497 \\ https://hal.science/hal-03395497}

Submitted on 22 Oct 2021

HAL is a multi-disciplinary open access archive for the deposit and dissemination of scientific research documents, whether they are published or not. The documents may come from teaching and research institutions in France or abroad, or from public or private research centers.
L'archive ouverte pluridisciplinaire HAL, est destinée au dépôt et à la diffusion de documents scientifiques de niveau recherche, publiés ou non, émanant des établissements d'enseignement et de recherche français ou étrangers, des laboratoires publics ou privés. 
Cette rubrique est dirigée par

M. VIART

Conservateur des eaux et forêts

Chef de la Division "Sylviculture

et aménagement des forêts "

Centre technique du génie rural des eaux et des forêts

Groupement de Nogent-sur-Vernisson

Domaine des Barres

45290 NOGENT-SUR-VERNISSON

\title{
ÉTUDE PRÉLIMINAIRE \\ DE L'INFLUENCE DE LA FERTILISATION SUR LA QUALITÉ DU BOIS DE CHÊNE
}

\author{
H. POLGE
}

Class, Oxford $815: 237.4: 174$ QUERCUS

Un article récemment publié dans cette revue (Garbaye et al., 1974) a rendu compte des premiers résultats obtenus dans des expériences de fertilisation réalisées en commun par la Station de recherches sur les sols forestiers et la fertilisation et la Station de sylviculture et de production du Centre national de recherches forestières en forêt de Bercé.

Trois dispositifs ont été implantés, désignés sous les noms de lieux-dits : "Route des Trembles », "Gaie Mariée » et "Rond du May ». C'est ce dernier, assis dans un perchis d'une trentaine d'années, qui a été retenu pour étudier les effets de la fertilisation sur certaines caractéristiques physiques du bois car :

— c'est celui où la fertilisation a été réalisée en premier lieu (1967 contre 1968 et 1969 pour les deux autres), ce qui constitue un avantage important puisqu'un des problèmes qui se pose est de savoir pendant combien de temps une éventuelle modification de la qualité du bois peut se manifester,

- c'est également celui où l'effet de la fertilisation sur la croissance a été le plus marqué, s'agissant d'un placeau installé sur sol lessivé podzolique de médiocre qualité, aussi bien au point de vue chimique qu'au point de vue physique.

L'étude envisagée ne devant avoir qu“un caractère exploratoire, il était normal de l'entreprendre dans ce dispositif où. selon toute probabilité, les effets de l'apport d'engrais sur les propriétés du bois devaient être les plus nets.

\section{ÉCHANTILLONNAGE}

Le dispositif du Rond du May comprend six placeaux :

- deux placeaux témoins non fertilisés (nos II et IV).

- deux placeaux avec fertilisation azotée et apport de chaux ( $n^{\circ s} V$ et $V I$ ),

- deux placeaux ayant reçu une fertilisation complète, plus un apport de chaux et de magnésium (nos I et III).

Les placeaux I, IV et VI ont fait l'objet, avant la saison de végétation 1972, d'une éclaircie relativement forte enlevant $30 \%$ de la surface terrière tout en régularisant la proportion de hêtres et la distribution des diamètres; dans les trois autres placeaux, au contraire, le nombre total de tiges existant au début de l'expérience a été conservé, à l'exception des plus gros hêtres qui gênaient la croissance du chêne. 
Toujours pour maximaliser les effets du traitement, on n'a retenu, pour l'étude de la qualité du bois, que les deux placeaux non fertilisés et les deux placeaux ayant reçu la fertilisation la plus forte.

Pour mieux rappeler les traitements qui leur sont appliqués, ces divers placeaux seront désignés dans les paragraphes suivants sous les références ci-dessous:

- T (témoin) pour le placeau II.

- TE (témoin éclairci) pour le placeau IV.

- F (fertilisé) pour la placeau III.

- FE (fertilisé éclairci) pour le placeau I.

Dans chaque placeau, l’échantillonnage a porté sur les 35 arbres dominants utilisés par la Station de sylviculture at de production pour les mesures dendrométriques.

\section{MATÉRIEL ET MÉTHODE}

Le matériel d'étude a consisté en carottes de sondage diamétrales de $5 \mathrm{~mm}$ prélevées de part en part à hauteur d'homme sur les 140 arbres de l'expérience (4 placeaux $\times 35$ ).

II s'agissait donc d'un échantillonnage non destructif et peu traumatisant, les trous ayant été rebouchés par des chevilles de bois imprégnées de sels de mercure et les orifices obturés à l'aide de mastic à graffer.

Les sondages ayant été effectués après la saison de végétation 1973 et la fertilisation avant la saison de végétation 1968, on disposait de 6 accroissements annuels pour étudier l'effet du traitement; les quatre placeaux en cause s'étant avérés assez différents les uns des autres avant même le début de l'expérience du point de vue des critères de qualité du bois, cela a été fait par comparaison avec les six années précédentes (1962 à 1967 inclusivement).

Chaque carotte diamétrale a été coupée en deux : Ia moitié côté entrée de la tarière, qui comporte toujours de petites distorsions dues à de légers mouvements de l'appareil lors de sa mise en place, a servi aux déterminations de densité globale; la moitié restante, toujours parfaitement rectiligne, a été utilisée pour les mesures de retraits et les études densitométriques.

La densité globale a été calculée sous forme d’infra-densité (rapport du poids anhydre au volume saturé) par une méthode maintenant classique faisant intervenir une saturation intégrale de l'échantillon.

Les retraits axiaux et tangentiels ont été mesurés entre l'état saturé et l'état stabilisé à $10 \%$ d'humidité relative à l'aide d'un capteur de déplacement électronique donnant une précision du micron ; les comparaisons ont porté sur les moyennes de toute une suite de mesures faites à des intervalles d'un quart de millimètre le long des échantillons, avec un minimum de 30 mesures pour chaque tranche de 6 accroissements annuels.

Les analyses densitométriques ont été réalisées à partir de radiographies tangentielles d'échantillons d'un millimètre d'épaisseur débités dans le côté sortie des carottes de sondage; de telles radiographies, qui donnent une image du bois dans un plan longitudinal radial, permettent dans le cas du chêne, il convient de le rappeler, de limiter les erreurs d'interprétation dues à la présence aléatoire de gros rayons ligneux ou de vaisseaux sur l'axe d'exploration (Polge, 1967).

Sur les courbes de variation de la densité du bois obtenues, ont été mesurées les densités minimales annuelles et les densités maximales annuelles (ce qui permet, par différence, de quantifier l'hétérogénéité du bois), ainsi que la pente moyenne des profils entre le début et la fin du bois d'été.

Les placeaux $F$ et FE d'une part, T et TE d'autre part, ne sauraient être considérés comme des répétitions du même traitement; ils différaient entre eux avant même le début de l’expérience, et l'opération d'éclaircie réalisée au cours de l"hiver 1971-1972 a abouti à appliquer un traitement différent à chacune des quatre parcelles unitaires. II n'est donc pas possible de procéder à une analyse statistique rigoureuse des résultats. Cependant, l'effet de l'éclaircie s'étant avéré minime sur la plupart des caractéristiques átudiées et n'ayant pu affecter que deux accroissements annuels sur les six qui se sont produits après l'année de la fertilisation, les résultats seront donnés par placeau, mais également en regroupant les deux placeaux fertilisés d'une part, et les deux placeaux témoins d'autre part.

Quant à l'influence de l'éclaircie, elle ne sera analysée que du point de vue de la caractéristique pour laqualle une séparation nette des années 1972 et 1973 a paru possible : la pente du bois d'été. 


\section{Infra-densité}

Les résultats sont donnés dans le tableau $\mathrm{n}^{\circ} 1 \mathrm{en} \mathrm{g} / \mathrm{dm}^{3}$ :

Tableau $n^{\circ} 1$

\begin{tabular}{|c|c|c|c|}
\hline & $1962-1967$ & $1968-1973$ & $\begin{array}{l}\text { Différences } \\
\text { entre périodes }\end{array}$ \\
\hline $\begin{array}{l}\text { Placeaux fertilisés }\left\{\begin{array}{llll}F & & & \\
F E & & . & \end{array}\right. \\
\text { Moyenne }\end{array}$ & $\begin{array}{l}605,0 \\
609,9 \\
607,5\end{array}$ & $\begin{array}{l}634,3 \\
641,3 \\
637,7\end{array}$ & $+30,2$ \\
\hline $\begin{array}{l}\text { Placeaux témoins }\left\{\begin{array}{lllll}T & & & & \\
T E & & & & \end{array}\right. \\
\text { Moyenne }\end{array}$ & $\begin{array}{l}616,0 \\
629,6 \\
622,8\end{array}$ & $\begin{array}{l}642,4 \\
648,7 \\
645,6\end{array}$ & $+22,8$ \\
\hline Test de comparaison de moyennes " $t$ " & & & $2,24^{+}$ \\
\hline
\end{tabular}

On voit qu'il existait des différences notables entre les placeaux avant même l'année de la fertilisation : la densité a augmenté entre la période 1962-1967 et 1968-1973, aussi bien dans les placeaux fertilisés que dans les témoins, mais l'augmentation a été plus marquée dans les premiers que dans les seconds, l'écart entre les différences relevées sur les valeurs moyennes des deux groupes de 70 arbres ayant reçu le même traitement initial étant significatif.

Cet effet défavorable de la fertilisation valait d'être noté, ne serait-ce qu'en raison du fait que la densité explique $64 \%$ des variations de la dureté (Polge et Keller, 1973); cependant, toutes les densités du tableau $n^{\circ} 1$ sont très élevées (en relation avec le jeune âge du peuplement) et les nuances que l'on peut relever dans la médiocrité du bois produit n'ont pour l'instant qu'une signification pratique limitée.

\section{Composantes de la densité}

L'évolution des composantes de la densité entre les six années qui ont précédé le début de l'expérience et les six années qui ont suivi se trouve résumée dans le tableau $n^{\circ} 2$ :

Tableau $n^{\circ} 2$

\begin{tabular}{|c|c|c|c|}
\hline & \multicolumn{3}{|c|}{$\begin{array}{c}\text { Différences entre les valeurs } 1968-73 \\
\text { et les valeurs } 1962-67\end{array}$} \\
\hline & $\begin{array}{l}\text { Densités maximales } \\
\text { annuelles }\end{array}$ & $\begin{array}{l}\text { Densités minimales } \\
\text { annuelles }\end{array}$ & $\begin{array}{l}\text { Hétérogénéité } \\
\text { (Maximum- } \\
\text { minimum) }\end{array}$ \\
\hline $\begin{array}{l}\text { Placeaux fertilisés }\left\{\begin{array}{l}F \\
F E \\
\text { Moyenne }\end{array}\right.\end{array}$ & $\begin{array}{l}-29,83 \\
-5,59 \\
-17,88\end{array}$ & $\begin{array}{l}-26,17 \\
-4,74 \\
-10,94\end{array}$ & $\begin{array}{l}-3,66 \\
-10,32 \\
-\quad 6,94\end{array}$ \\
\hline $\begin{array}{l}\text { Placeaux témoins }\left\{\begin{array}{l}T \\
T E \\
\text { Moyenne }\end{array}\right] \\
\text {. }\end{array}$ & $\begin{array}{r}-3,49 \\
0,12 \\
-\quad 1,71\end{array}$ & $\begin{array}{l}-12,26 \\
-17,88 \\
-15,03\end{array}$ & $\begin{array}{r}8,77 \\
18,00 \\
13,32\end{array}$ \\
\hline « $t »$ entre fertilisés et témoins. & $2,56^{+}$ & $0,37^{\mathrm{NS}}$ & $1,62^{\mathrm{NS}}$ \\
\hline
\end{tabular}


On observe tout d'abord que, aussi bien densités maximales annuelles que minimales, ont diminué entre la première et la seconde des deux périodes de six ans considérées; ceci pourrait surprendre puisque l'infra-densité a de son côté augmenté, mais l'examen des enregistrements densitométriques montre une augmentation avec l'âge du pourcentage de bois final de densité élevée qui a pu compenser, et audelà, l'effet de la diminution des densités maximales et minimales annuelles.

Dans la comparaison entre traitements, une différence significative apparaît en ce qui concerne la densité maximale annuelle qui a fortement diminué dans les arbres fertilisés, alors qu'elle est restée pratiquement inchangée dans les témoins.

Quant à l'hétérogénéité, elle s'est abaissée de façon sensible dans les arbres fertilisés et aggravée au contraire dans les témoins, mais du fait de la très grande dispersion des valeurs individuelles, les deux populations ne se différencient pas l'une de l'autre de façon significative.

\section{Pente du bois final}

L'examen visuel des radiographies axiales, confirmé par l'observation des profils densitométriques des radiographies tangentielles, a montré que les variations de la structure du bois d'été, et corrélativement celles de la densité, étaient, des divers critères étudiés, celui qui traduisait le mieux la réaction des arbres aux traitements; on s'est donc efforcé de quantifier de façon objective cette donnée en adoptant sur les profils densitométriques, comme début du bois d'été, le sommet du premier pic suivi d'une baisse de densité représentée par une chute d'au moins $1 \mathrm{~cm}$ (traduisant une différence de $85 \mathrm{~g} / \mathrm{dm}^{3}$ ) et d'une largeur minimale de $1 \mathrm{~mm}\left(1 / 5^{\mathrm{e}}\right.$ de $\mathrm{mm}$ vraie grandeur $)$; quant à la fin du même bois d'été, elle correspond de façon conventionnelle au sommet du dernier pic précédant une partie de courbe ayant une pente d'au moins $1 / 2 \mathrm{~mm}$ dans le sens des abscisses, sur $1 \mathrm{~cm}$ dans le sens des ordonnées.

Sur la figure $n^{\circ} 1$ sont repérées, à l'aide de cercles, les limites ainsi définies pour un certain nombre d'accroissements annuels caractéristiques (les arbres en cause sont assez représentatifs de la moyenne des placeaux au point de vue largeur des cernes, mais pas nécessairement au point de vue densité du bois).

Une analyse de variance entre placeaux a montré qu'il n'existait pas de différences globales pour la période $1962-1967$ ( $F=2,22$ avec 3 et 136 degrés de liberté), mais des différences très significatives au seuil de $1 \%$ pour la période $1968-1971(F=16,85)$ et pour la période 1972-1973 ( $F=9,70)$.

Un test de comparaison de moyennes multiples a donné les résultats portés sur le tableau $n^{\circ} 3$ :

Tableau $\mathrm{n}^{\circ} 3$

\begin{tabular}{|c|c|c|c|c|c|}
\hline Périodes & & & & & \\
\hline $1962-1967$ & $\begin{array}{l}\text { Placeaux } \\
\text { Pentes moyennes }\end{array}$ & $\begin{array}{c}\text { TE } \\
-4,60\end{array}$ & $\begin{array}{l}\text { FE } \\
-3,70\end{array}$ & $\begin{array}{c}\mathrm{T} \\
-3,47\end{array}$ & $\begin{array}{c}F \\
-3,32\end{array}$ \\
\hline $1968-1971$ & $\begin{array}{l}\text { Placeaux } \\
\text { Pentes moyennes }\end{array}$ & $\begin{array}{c}\mathrm{TE} \\
-3,69 \\
\end{array}$ & $-2,68$ & $\begin{array}{c}\mathrm{FE} \\
-1,51\end{array}$ & $\stackrel{F}{-1,17}$ \\
\hline \multirow[t]{2}{*}{$1972-1973$} & $\begin{array}{l}\text { Placeaux } \\
\text { Pentes moyennes }\end{array}$ & $\begin{array}{c}\mathrm{T} \\
-3,70\end{array}$ & $\begin{array}{l}\text { TE } \\
-2,85\end{array}$ & $\begin{array}{c}\mathbf{F} \\
-2,01\end{array}$ & $\begin{array}{c}\mathrm{FE} \\
-1,54\end{array}$ \\
\hline & & & & 1 & 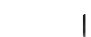 \\
\hline
\end{tabular}

Conformément aux usages, les valeurs regroupées à l’intérieur d’une même accolade ne diffèrent pas significativement entre elles au seuil de $5 \%$.

On peut faire les observations suivantes:

La pente a diminué pour tous les placeaux entre 1962-1967 et 1968-1971, ce qui traduit un gradient normal de variation avec l'âge; cependant, si on met à part le placeau TE qui se différenciait déjả des 

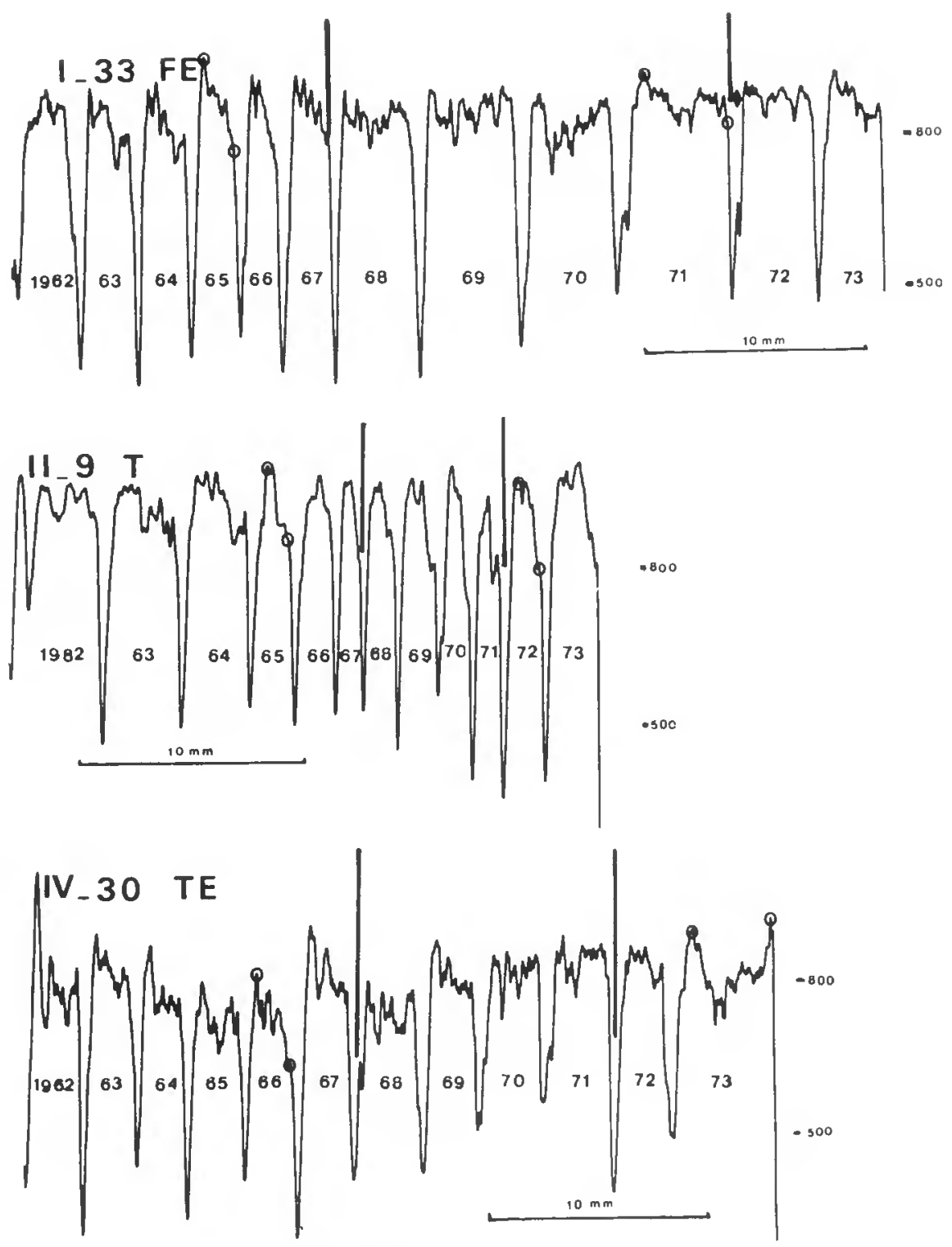

Figure no 1 - Profils densitométriques d’échantillons-types.

trois autres avant traitement, on voit que les deux placeaux fertilisés, qui ne se distinguaient pas du placeau T à l'origine, s'en écartent significativement après l'apport d'engrais dont ils ont bénéficié.

Après l'éclaircie, les positions relatives à l'intérieur des deux groupes (fertilisés et témoins) se trouvent inversées, le traitement sylvicole ayant, comme précédemment la fertilisation, entraîné une baisse de la pente du bois d'été pour les placeaux FE et TE.

Les exemples de la figure $n^{\circ} 1$ illustrent bien ces différentes évolutions : l’arbre témoin, ni fertilisé, ni éclairci, ( $n^{\circ} 9$ du placeau T) a un bois d’été présentant toujours une pente négative importante.

Sur le profil de l'arbre 33 du placeau FE, on voit l'effet spectaculaire de la fertilisation sur la largeur des accroissements avec, corrélativement, une diminution très importante de la pente du bois d'été. 


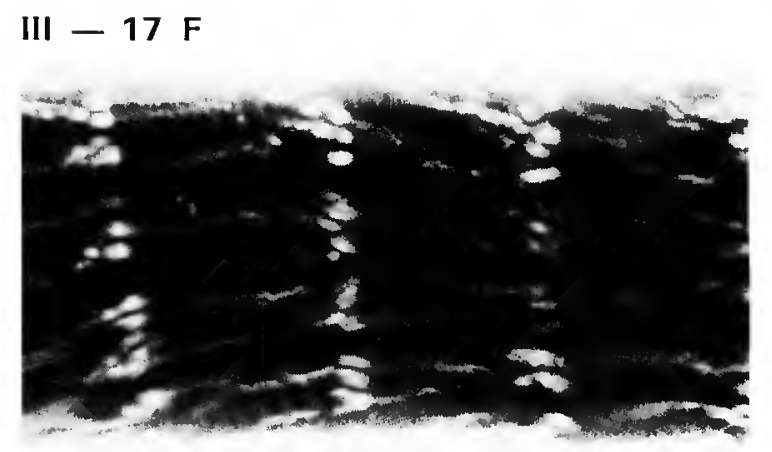

Figure no 2 - Positifs de radiographie d'un échantillon fertilisé (à la partie supérieure) et d'un témoin montrant la répartition différente des fibres dans la zone de bois final.

\section{$V-33$ TE}

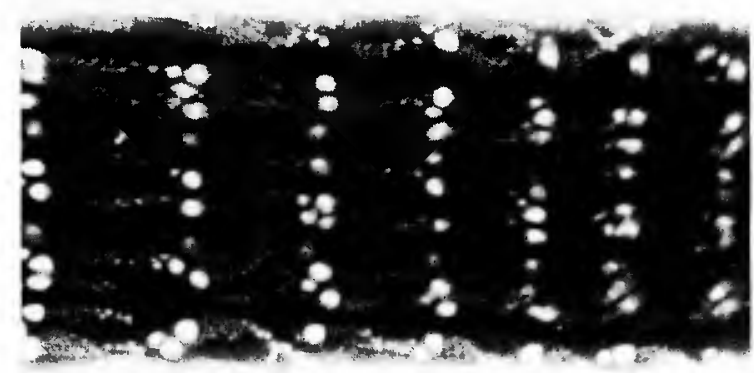

Quant à l'arbre 30 du placeau TE, qui n'a bénéficié que de l'éclaircie, il a réagi, avec quelque retard, à celle-ci en formant en 1973 un accroissement bien plus fort que les précédents, mais avec une pente du bois d'été, non seulement réduite, mais même devenant au caś particulier positive.

Ces variations de pente du bois final résultent de la modification de la répartition des différents types d'éléments anatomiques entraînée par la fertilisation ou par l'éclaircie.

Dans les témoins (voir par exemple quelques accroissements avant éclaircie de l'arbre 33 du placeau TE sur la figure $n^{\circ} 2$ ), le bois final comporte des plages triangulaires de fibres à densité élevée dont la base se situe immédiatement après la fin de la zone poreuse; les intervalles compris entre les sommets de ces triangles sont remplis par des zones de petits vaisseaux et de parenchyme périvasculaire de densité beaucoup plus faible, ce qui se traduit par une diminution de la densité d'une extrémité à l'autre de la zone de bois final.

Dans les arbres fertilisés (arbre 17 du placeau F), les zones fibreuses ont une forme à peu près rectangulaire, leur largeur variant assez peu tout au long de la période de formation du bois d'été; il en est automatiquement de même pour les plages à faible densité qui les séparent, en sorte qu'au total, la densité reste sensiblement constante à l'intérieur du bois final

\section{Rétractibilité}

L'évolution des retraits tangentiels et axiaux au cours de l'expérience (différences entre les valeurs 1968-1973 et les valeurs 1962-1967) se trouve résumée dans le tableau n 4 :

On constate que le retrait tangentiel a diminué davantage dans le bois des arbres témoins que dans celui des fertilisés; la différence entre traitements, bien que très importante, n'est cependant pas significative au seuil de $5 \%$ en raison de la forte variabilité individuelle de la caractéristique en cause.

Quant au retrait axial, il s'est aggravé dans les arbres fertilisés alors qu'il diminuait très fortement dans les témoins, la différence entre les uns et les autres étant statistiquement significative. 
11 convient cependant de noter qu'en valeur absolue cette rétractibilité n'est jamais très élevée; elle est passée pour les fertilisés de $0,069 \%$ à $0,091 \%$, et pour les témoins de $0,174 \%$ à $0,102 \%$, alors qu'elle ne présente des inconvénients sérieux, et encore pour des pièces de grande longueur, qu'au-delà de $0,2 \%$ environ.

Tableau $n^{\circ} 4$

\begin{tabular}{|c|c|c|c|c|}
\hline & \multicolumn{2}{|c|}{ Retrait tangentiel } & \multicolumn{2}{|c|}{ Retrait axial } \\
\hline & $\begin{array}{l}\text { Valeurs } \\
\text { par placeaux }\end{array}$ & $\begin{array}{l}\text { Valeurs } \\
\text { moyennes }\end{array}$ & $\begin{array}{l}\text { Valeurs } \\
\text { par placeaux }\end{array}$ & $\begin{array}{l}\text { Valeurs } \\
\text { moyennes }\end{array}$ \\
\hline Placeaux fertilisés $\left\{\begin{array}{l}F \\
F E\end{array}\right.$ & $\begin{array}{l}-0,30 \\
-0,34\end{array}$ & $-0,324$ & $\begin{array}{l}+0,073 \\
-0,029\end{array}$ & $+0,0221$ \\
\hline Placeaux témoins $\left\{\begin{array}{l}T \\
T E\end{array}\right.$ & $\begin{array}{l}-0,68 \\
-0,43\end{array}$ & $-0,558$ & $\begin{array}{l}-0,087 \\
-0,064\end{array}$ & $-0,072$ \\
\hline $\begin{array}{l}\text { Test « } t \text { " de comparaison } \\
\text { de moyennes . . . . . . }\end{array}$ & & $1,82^{\mathrm{NS}}$ & & 2,34 \\
\hline
\end{tabular}

\section{CONCLUSION}

Malgré la faible taille des échantillons et le petit nombre d'années étudiées, les essais de caractère exploratoire dont il est rendu compte ici ont permis de dégager des tendances et, dans certains cas, de faire apparaitre des affets significatifs des traitements.

Du point de vue de la qualité du bois, et pour autant que l'on envisage des utilisations nobles, en particulier comme bois de tranchage, l'influence de la fertilisation s'exerce en général dans un sens défavorable puisqu'elle entraine une augmentation significative de la densité globale et une évolution peu satisfaisante de la rétractibilité dans le sens axial.

L'étude de la pente du bois final fournit une explication à ce double phénomène puisque densité et retrait sont liés à la proportion de fibres à la fin de la zone de bois d'été, qui est effectivement plus impor tante dans les arbres fertilisés; elle a également permis de voir que les augmentations de largeurs de cernes, dues à l'éclaircie d'une part, et à la fertilisation d'autre part, se traduisaient par des effets analogues sur la structure du bois, ce qui semble logique, mais il était cependant bon de le vérifier par l'expérience.

On peut noter également que les perchis de Bercé réagissent à une accélération de croissance, quelle qu'en soit l'origine, de façon très comparable aux vieux arbres de Tronçais étudiés par ailleurs (Polge et Keller, 1973).

Le seul avantage technologique de la fertilisation a trait à la baisse de densité maximale annuelle qu'elle occasionne et qui, à son tour, entraîne une réduction de l'hétérogénéité. Le phénomène présente cependant une amplitude insuffisante pour contrebalancer les influences en sens contraire mises en évidence en ce qui concerne la densité globale, la rétractibilité et la structure du bois d'été.

Enfin, si l'étude n’a pas été faite année par année, il résulte néanmoins de l'examen des enregistrements de la densité du bois que dans tous les échantillons où l'effet de l'apport d'engrais sur la largeur des accroissements subsiste six ans après le traitement, il en est de même pour celui qui en résulte sur la qualité du bois.

Au total, l'expérience de Bercé montre que le gain de croissance assez spectaculaire occasionné par l'apport d'engrais s'accompagne malheureusement d'une certaine tendance à une baisse de qualité; cette constatation ne doit pas conduire à une condamnation de la fertilisation sur des jeunes peuplements de chêne car les arbres en cause n'ont pas encore atteint un diamètre tel que la production de bois de tranchage se trouve compromise, et en outre l'évolution défavorable de certains critères de valeur technologique ne présente pas pour l'instant un caractère dramatique. 


\section{H. POLGE}

II convient cependant de rester vigilant puisque l'influence des engrais semble s'exercer pendant de nombreuses années après traitement, et une nouvelle étude, complétée si possible par des essais sur éprouvettes normalisées, devrait donc être entreprise à un âge plus avancé et dans un dispositif permettant une meilleure interprétation statistique des résultats.

II resterait également à expliquer les effets technologiques de la fertilisation. Le premier facteur limitant de la croissance du chêne, avant même la nutrition minérale, étant l'approvisionnement en eau (Leroy, 1967) on aurait pu penser qu'un apport d'engrais aurait surtout profité au bois initial formé à une période de l'année où l'eau ne fait pas défaut dans le secteur ligérien; il n’en est apparemment rien. Ceci pourrait tenir au fait que dans les feuillus à zone poreuse, au contraire de ce qui se passe pour ceux à pores diffus, l'assise cambiale commence à fonctionner avant le débourrement et peut même produire des vaisseaux en cas d'ébourgeonnement total; son activation initiale serait donc due à des substances de croissance préexistantes ou synthétisées à ce niveau et pourrait par suite dépendre de facteurs biotiques intervenus antérieurement.

\section{REMERCIEMENTS}

M. Hoyau, Chef du Centre de Gestion de I'O.N.F., a bien voulu autoriser les prélèvements d'échantillons nécessaires à cette étude.

MM. Oswald et Garbaye, Chargés de recherches au C.N.R.F., ont fait du manuscrit une critique très constructive.

De nombreux techniciens de la Station de recherches sur la qualité des bois et de la Station de biométrie ont collaboré, soit aux mesures brutes des données, soit à leur traitement.

Que tous soient remerciés pour leur précieux concours.

\section{BIBLIOGRAPHIE}

GARBAYE (J.), LEROY (Ph.), OSWALD (H.). - Premiers résultats de cinq années de fertilisation sur jeunes peuplements de chêne en forêt de Bercé. Revue forestière française, no 1, 1974, pp. 51-58.

LEROY (Ph.). - Variations de productivité du chêne sur les pentes à Bercé. Nécessité d'une cartographie des sols. Revue forestière française, no 10, 1967, pp. 568-612.

POLGE (H.). - Propositions pour une meilleure utilisation des courbes de variations de la densité. XIV IUFROKongress, Section 41, septembre 1967, pp. 286-299.

POLGE (H.), KELLER (R.). - Qualité du bois et largeurs d'accroissements en forêt de Tronçais. Annales des sciences forestières, vol. $30, n^{\circ} 2,1973$, pp. 91-126. 